\title{
17 $\alpha$-HYDROXYPROGESTERONE AND 21-DESOXYHYDROCORTISONE; THEIR METABOLISM AND POSSIBLE ROLE IN CONGENITAL ADRENAL VIRILISM ${ }^{1}$
}

\author{
By JOSEPH W. JAILER, JAY J. GOLD, RAYMOND VANDE WIELE, AND \\ SEYMOUR LIEBERMAN \\ (From the Departments of Medicine and Obstetrics and Gynecology, College of Physicians and \\ Surgeons, Columbia University and the Presbyterian Hospital, New York, N. Y.)
}

(Submitted for publication June 13, 1955; accepted July 7, 1955)

Although virilism due to bilateral adrenal hyperplasia is a relatively rare condition, this syndrome has stimulated much interest and research. Previous work has demonstrated that the adrenals of patients with this disease respond differently from the normal when they are further stimulated with exogenous ACTH. Lewis and Wilkins (1), Bartter and his associates (2), and Jailer, Louchart, and Cahill (3) have shown that although the administration of ACTH to patients with congenital adrenal hyperplasia results in a further increase in the already elevated urinary 17-ketosteroids, other responses are different from those seen in normal subjects. For example, following the administration of ACTH to patients with adrenal virilism there is no appreciable rise in urinary corticoids, measured as reducing or formaldehydogenic steroids, no decrease in the circulating eosinophils, and no sodium retention. Furthermore, Kelley, Ely, and Raile (4), and then subsequently Bayliss, Broadbent, and Steinbeck (5), Bongiovanni, Eberlein, and Cara (6) and Christy, Wallace, and Jailer ( 7 ) have shown that there is no increase in plasma corticoids in the great majority of these patients under these circumstances.

That the administration of cortisone or hydrocortisone to patients with congenital virilism results in a fall in the urinary 17-ketosteroids and a regression in the characteristic virilizing symptoms of this disease was first demonstrated by Wilkins, Lewis, Klein, and Rosemberg (8) and confirmed by others $(2,9,10)$. It is generally accepted that this effect of cortisone is mediated through the inhibition of ACTH secretion by the pituitary.

The underlying steroidal pathology in the adrenals of these patients has been the subject of some speculation. Bartter, Albright, Forbes, Leaf,

\footnotetext{
1 Aided by Grants from the National Institutes of Health, The U.S.P.H.S., and the Upjohn Company.
}

Dempsey, and Carroll (2) have postulated that this condition is characterized by an increased secretion of adrenal androgen(s) and a decreased production of hydrocortisone. Consequently, an increased amount of $\mathrm{ACTH}$ would be required to stimulate the adrenal to secrete the normal amount of hydrocortisone needed for homeostasis. Excessive amounts of circulating ACTH have been found in the blood of such patients (Sydnor, Kelley, Raile, Ely, and Sayers [11]). Apparently, the excessive amounts of adrenal androgen(s) are incapable of inhibiting ACTH secretion. Jailer (12) suggested that the defect in this syndrome may reside in the enzymatic hydroxylation mechanisms which had previously been shown by Hechter, Zaffaroni, Jacobsen, Levy, Jeanloz, Schenker, Pincus, Hayano, and Dorfman (1315) to be essential for the biosynthesis of Compounds $B$ and $F$ from simpler steroidal intermediates. This group had previously demonstrated that surviving adrenal tissue possesses hydroxylases which are capable of introducing hydroxyl groups into certain C-21 steroids at carbons 11 , 17 and 21 , to yield the adrenal hormones: corticosterone and hydrocortisone (15). The simplest C-21 precursor which can be hydroxylated in this fashion may be pregnenolone, a substance which has been proven to be an intermediate in the formation of these hormones from cholesterol (16, 17).

The possibility that one of the important metabolic defects in congenital adrenal hyperplasia may be the inability of the adrenal gland to accomplish the complete enzymatic hydroxylation of the steroidal precursors has been suggested (12). Such a failure might result in the formation of partially hydroxylated secretory products, some of which could be androgenic in nature or others which could be converted to androgens during catabo- 
TABLE I

Urinary metabolites identified in patients with congenital adrenal hyperplasia

\begin{tabular}{|c|c|c|c|c|c|c|c|c|c|}
\hline \multirow[b]{2}{*}{ Steroid } & \multicolumn{9}{|c|}{ Mg./day } \\
\hline & J. B. & R. K. & D. P. & E. B. & S. B. & L. Y. & R. E. & S. P. & J. L. \\
\hline \multirow{6}{*}{$\begin{array}{l}\text { Dehydroisoandrosterone } \\
\text { Androsterone } \\
\text { Etiocholanolone } \\
11 \text { Keto-etiocholanolone } \\
11 \beta \text {-hydroxyandrosterone } \\
11 \beta \text { hydroxy-etiocholanolone } \\
\text { Pregnanolone } \\
11 \text { Keto-pregnanolone } \\
17 \alpha \text { hydroxy-pregnanolone }\end{array}$} & \& & \& & 9 & $\sigma^{7}$ & 9 & $\sigma^{\pi}$ & 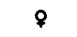 & q & \multirow{6}{*}{$\begin{array}{r}9 \\
7.8 \\
12.7 \\
3.9 \\
3.9\end{array}$} \\
\hline & & & 9.1 & & & & & & \\
\hline & $\begin{array}{l}3.8 \\
2.1\end{array}$ & $\begin{array}{l}4.0 \\
2.1\end{array}$ & $\begin{array}{l}4.4 \\
5.6\end{array}$ & $\begin{array}{r}10.8 \\
4.2\end{array}$ & $\begin{array}{l}3.2 \\
1.6\end{array}$ & 3.9 & 10.8 & $\begin{array}{l}3.2 \\
0.6\end{array}$ & \\
\hline & $\begin{array}{l}2.1 \\
1.7\end{array}$ & & & & $\begin{array}{l}1.0 \\
1.8\end{array}$ & & 1.8 & & \\
\hline & 2.3 & 4.0 & $\begin{array}{c}6.7 \\
\text { trace }\end{array}$ & 7.3 & 2.0 & 1.7 & & & \\
\hline & $\begin{array}{l}1.6 \\
6.9\end{array}$ & $\begin{array}{l}3.1 \\
5.9\end{array}$ & & 5.9 & 2.2 & $\begin{array}{r}1.8 \\
17.0 \\
2.9\end{array}$ & 4.6 & $\begin{array}{l}2.1 \\
1.2\end{array}$ & \\
\hline
\end{tabular}

lism. Two such intermediary steroids, $17 \alpha$-hydroxyprogesterone and 21-desoxyhydrocortisone, have been implicated in this syndrome (12). In this study a comparison of the urinary steroidal metabolites resulting from the exogenous administration of $17 \alpha$-hydroxyprogesterone and 21-desoxyhydrocortisone with those steroids known to be found in the urine of patients with untreated congenital adrenal hyperplasia was made.

Pfiffner and North (18) have isolated $17 \alpha$-hydroxyprogesterone from extracts of adrenal tissues and demonstrated that it is androgenic. Gassner (19) has recently reassayed this steroid and found that while it was not active when applied locally to the cock's comb it has about one-half the androgenic activity of methyltestosterone when it is administered orally to cockerels. The administration of $17 \alpha$-hydroxyprogesterone to patients (20) and to rats (21) results in an increase in the urinary excretion of neutral 17 -ketosteroids. It has been shown by Fukushima, Dobriner, and Gallagher (22), by means of deuterium-labeled $17 \alpha$ hydroxyprogesterone, that this adrenal product is metabolized to androsterone and etiocholanolone. Bongiovanni, Eberlein, and Cara (23) have demonstrated that $17 \alpha$-hydroxyprogesterone is also metabolized to pregnane-3 $\alpha, 17 \alpha, 20 \alpha$-triol, a steroid characteristically found in the urine of patients with adrenal virilism $(6,24)$.

21-Desoxyhydrocortisone, another possible partially hydroxylated intermediate of adrenal hormone biosynthesis, has not as yet been associated with any biological activity, nor has its metabolism been previously investigated. The urinary products resulting from the administration of the closely related 21-desoxycortisone have been stud- ied by Burstein, Savard, and Dorfman (25) who have administered it to two patients with rheumatoid arthritis and have recovered from the urine 11-keto-etiocholanolone, $11 \beta$ hydroxy-etiocholanolone, 11 $\beta$-hydroxyandrosterone and 21-desoxytetra-hydrocortisone.

The objective in this investigation was to determine whether the urinary products resulting from the administration of $17 \alpha$-hydroxyprogesterone and 21-desoxyhydrocortisone are similar to and could account for the known urinary metabolites found in adrenal virilism. For comparison, the endogenously excreted steroids in the urine of nine untreated patients with congenital adrenal hyperplasia were determined by essentially identical methods (Table I). In addition to the compounds identified in Table I, others have reported the isolation of pregnane $3 \alpha, 20 \alpha$, diol ; pregnane$3 \alpha, 17 \alpha, 20 \alpha$-triol (26) and pregnanetriol-11-one (reviewed in 27), compounds which were not detected by the techniques employed in this study.

\section{METHODS AND MATERIALS}

Patients who were chosen for the study of the metabolism of exogenously administered steroids excreted but minimal amounts of endogenous 17 -ketosteroids.

$17 \alpha-\mathrm{Hydroxyprogesterone}{ }^{2}$ was administered orally to eight patients as a water suspension or in gelatine capsules. Each patient received a total of between 600 and $1,000 \mathrm{mg}$. in divided doses over a two or three-day period. The patients used were as follows:

1. A 40-year-old female Addisonian patient maintained on $25 \mathrm{mg}$. a day of oral cortisone (F. S.).

2. A 55-year-old man with hypopituitarism (S. W.).

2 The $17 \alpha$-hydroxyprogesterone was kindly supplied by the Syntex Corporation and the 21-desoxyhydrocortisone by the Upjohn Company. 
3. A 21-year-old male Addisonian patient whose only replacement therapy consisted of implanted pellets of DCA (J. R.).

4. A 55-year-old man with hypopituitarism who received $15 \mathrm{mg}$. a day of thyroid extract (G. S.).

5. An 11-year-old female pseudohermaphrodite whose urinary 17 -ketosteroids were being suppressed by means of daily doses of $50 \mathrm{mg}$. of parenteral cortisone (F. K.).

6. A 17-year-old female pseudohermaphrodite whose therapeutic regimen consisted of $100 \mathrm{mg}$. of parenteral cortisone administered thrice weekly (J. L.).

7. A 10-year-old female pseudohermaphrodite who received $100 \mathrm{mg}$. of cortisone parenterally thrice weekly in order to maintain her urinary $17-$ ketosteroids close to normal (R. E.).

8. A 6-year-old female pseudohermaphrodite who was receiving $100 \mathrm{mg}$. per day of parenteral cortisone to suppress her 17 -ketosteroids (S. P.).

21-Desoxyhydrocortisone was administered to two patients, both of whom received $300 \mathrm{mg}$. daily for three days by the oral route. L. B. was a 49-year-old male with hypoadrenalism and hypothyroidism, and $H$. $H$. was a 41-year-old housewife who had undergone bilateral adrenalectomy previously because of Cushing's syndrome due to bilateral adrenal hyperplasia. She required 25 mg. per day of oral cortisone for maintenance. The latter patient also received $17 \alpha$-hydroxyprogesterone several days prior to the administration of 21-desoxyhydrocortisone.

The total neutral urinary 17-ketosteroids were determined by the method of Holtorff and Koch (28) after acid hydrolysis on aliquots of complete 24-hour urine specimens. Another aliquot of urine was hydrolyzed with glucuronidase and the method of Sommerville, Marrian, and Kellar (29) employed to estimate the amounts of pregnanediol. This method probably determines pregnanetriol as well (23).

Still another portion (approximately 1 liter) of some of the 24-hour urines was processed in the following manner: The urine was acidified to $2 \mathrm{~N}$ with 50 per cent sulfuric acid and then extracted with ether for 24 hours in a continuous extractor. The ether extract was washed with saturated $\mathrm{Na}_{2} \mathrm{CO}_{3}, 10$ per cent $\mathrm{NaOH}$ and water until neutral and then evaporated to dryness. The above alkaline washings were added to the residual urine and the mixture treated with ten volumes per cent of hydrochloric acid. The mixture was then boiled for $20 \mathrm{~min}$ utes and extracted continuously a second time with ether for 24 hours. The ether extract was treated as above and combined with the first ether extract. The dried combined extract was dissolved in $10 \mathrm{ml}$. benzene and chromatographed on an alumina column. In the first several chromatograms elution was carried out by the Dingemanse method (30) and later by the gradient elution method of Lakshmanan and Lieberman (31). Zimmermann reactions were performed on $1 \mathrm{ml}$. aliquots of the eluates and the results plotted ( $\mathrm{mcg}$. per $\mathrm{ml}$.) against the fraction number. The 17 -ketosteroids resolved by the chromatographic separation were identified by means of infrared spectrometry.

\section{RESULTS}

\section{Congenital adrenal hyperplasia}

The amounts of the urinary metabolites found in a 24-hour urine specimen from each of nine untreated patients with congenital adrenal hyperplasia, ranging in age between 5 and 17 years are shown in Table I.

\section{$17 \alpha$-Hydroxyprogesterone}

The extract from at least one control urine from each patient who subsequently received this substance was resolved by chromatographic analysis. In only two cases were any ketosteroids identifiable. In J. L., $0.7 \mathrm{mg}$. 11 $\beta$-hydroxyandrosterone was found and in S. P., $1.8 \mathrm{mg}$. 11 $1 \beta$-hydroxyandrosterone, $0.8 \mathrm{mg}$. 11-keto-etiocholanolone and $1.3 \mathrm{mg} .17 \alpha$-hydroxy-pregnanolone were found.

TABLE II

Effect of 17 $\alpha$-hydroxyprogesterone on the urinary excretion of neutral $17 K S$ and "pregnanediol"

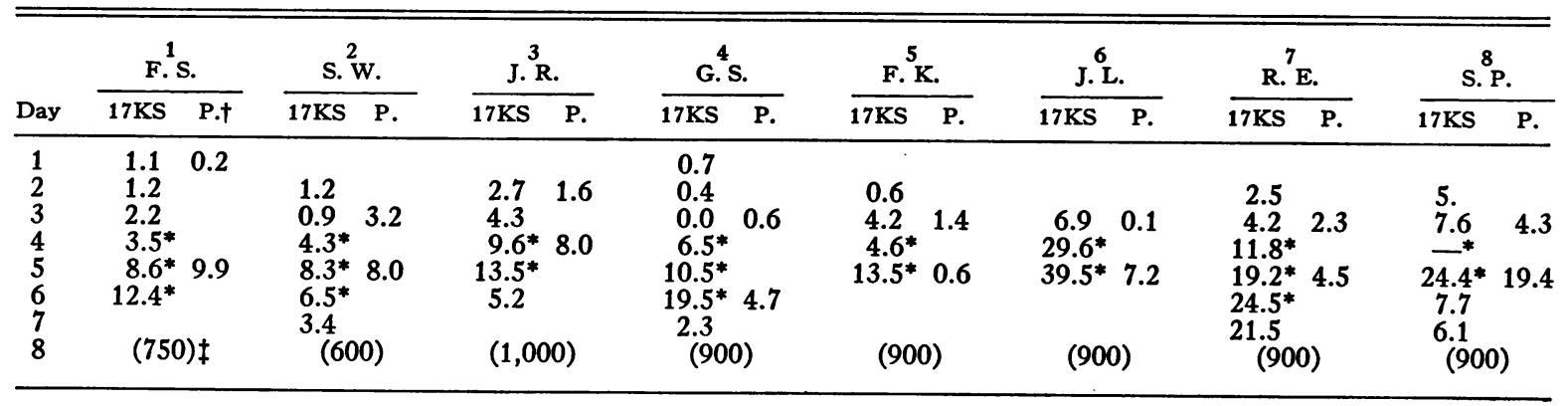

* Day $17 \alpha$-hydroxyprogesterone was administered.

$\dagger$ P. = "Pregnanediol" as measured by the Sommerville-Marrian method.

¥ Figure in parentheses-total mg. administered. 
TABLE III

The effect of orally administered $17 \alpha$-hydroxyprogesterone and 21-desoxyhydrocortisone on urinary $17 K S$

\begin{tabular}{|c|c|c|c|c|}
\hline \multirow[b]{2}{*}{ Day } & \multicolumn{2}{|r|}{ H. H. } & \multicolumn{2}{|r|}{ L. B. } \\
\hline & $17 \mathrm{KS}$ & Treatment & $17 \mathrm{KS}$ & Treatment \\
\hline $\begin{array}{r}1 \\
2 \\
3 \\
4 \\
5 \\
6 \\
7 \\
8 \\
9 \\
10 \\
11 \\
12\end{array}$ & $\begin{array}{r}4.4 \\
6.9 \\
17.9 \\
45.8 \\
55.6 \\
22.1 \\
19.5 \\
30.6 \\
26.6 \\
25.2 \\
10.2 \\
8.2\end{array}$ & 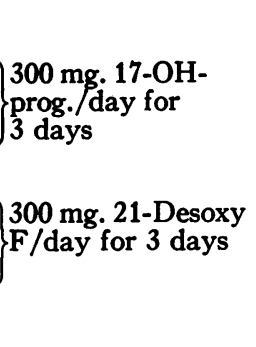 & $\begin{array}{r}4.0 \\
5.0 \\
5.9 \\
7.0 \\
19.4 \\
32.2 \\
20.8 \\
9.3 \\
5.7 \\
3.8\end{array}$ & $\left\{\begin{array}{l}300 \text { mg. 21-Desoxy } \\
\text { F/day for } 3 \text { days }\end{array}\right.$ \\
\hline
\end{tabular}

The oral administration of $17 \alpha$-hydroxyprogesterone resulted in an increase in the total neutral urinary 17-ketosteroids in all patients (Tables II and III).

Urinary extracts obtained from each patient during the treatment period were also analyzed. Androsterone and etiocholanolone were invariably found. The percentage yields were not easily determined since only one day's urine was analyzed rather than a pool of all the urines excreted during the treatment period. Nevertheless, the data permit the observation of an approximate ratio of about $1: 5$ of androsterone to etiocholanolone ( $5 \alpha-$ $5 \beta$ ). In contrast to the above, the urines collected for the three days while the patient, H. H., was receiving $17 \alpha$-hydroxyprogesterone were pooled and analyzed (Table III). Five and eight-tenths mg. of androsterone and $26.1 \mathrm{mg}$. etiocholanolone were found during this period, whereas no urinary metabolites could be identified during the control period.
The administration of $17 \alpha$-hydroxyprogesterone also resulted in an increased excretion of the "pregnanediol-pregnanetriol complex" as measured by the Sommerville, Marrian, and Kellar method (29), Table II. The presence of both pregnanediol and pregnanetriol in this fraction was suggested when the chromatographic method of Bongiovanni and Clayton (32) was employed and the sulfuric acid chromogenic absorption curves were compared to those of standard compounds. In one patient (S. W.) for example, the pregnanediol rose from $1.8 \mathrm{mg}$. to $19.4 \mathrm{mg}$. and the pregnanetriol value from 1.8 to $15.0 \mathrm{mg}$. after the administration of $17 \alpha$-hydroxyprogesterone.

In addition, $3 \alpha$-hydroxy-pregnan-20-one was found in the urine of one patient and $17 \alpha$-hydroxypregnanolone in the urine of four patients who had received $17 \alpha$-hydroxyprogesterone. The latter metabolite was identified by means of infrared spectrometry and the former by means of infrared spectrometry, melting point and mixed melting point.

\section{1-Desoxyhydrocortisone}

Two patients received $300 \mathrm{mg}$. per day for three days of 21-desoxyhydrocortisone by mouth. This regimen resulted in an appreciable rise in the urinary excretion of the total neutral 17 -ketosteroids (Table III).

In spite of the Zimmermann chromogen found during the control period in the urines of $\mathrm{H}$. $\mathrm{H}$., and L. B., no identifiable ketosteroids were found in the urine of $\mathrm{H}$. H., whereas but a fraction of a milligram of etiocholanolone was detected in the control urine of $\mathrm{L}$. B.

TABLE IV

The urinary metabolites isolated after the administration of 21-desoxyhydrocortisone

\begin{tabular}{|c|c|c|c|}
\hline L. B. & mg. & H. H. & $m g$. \\
\hline 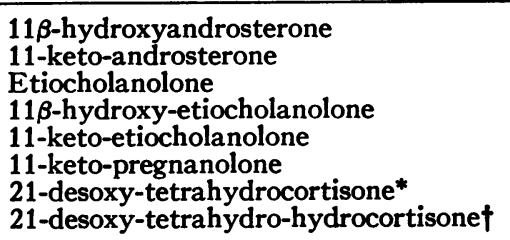 & $\begin{array}{r}20.5 \\
20.7 \\
4.3 \\
12.2 \\
16.3 \\
7.8\end{array}$ & $\begin{array}{l}11 \beta \text {-hydroxyandrosterone } \\
11 \text { keto-etiocholanolone } \\
11 \text { keto-pregnanolone }\end{array}$ & $\begin{array}{r}9.5 \\
11.5 \\
11.6\end{array}$ \\
\hline
\end{tabular}

* $3 \alpha, 17 \alpha$ dihydroxy-pregnane 11,20 dione.

$+3 \alpha, 11 \beta, 17 \alpha$ trihydroxy-pregnan-20-one. 
The urinary metabolites found as a result of the administration of the test substance are shown in Table IV. 11-Keto-pregnanolone was identified by melting point and mixed melting point as well as infrared spectrometry. Aliquots of urine from the control period and during the time patient L. B. was receiving 21-desoxyhydrocortisone were analyzed by the method of Zondek and Finkelstein (33) for neutral fluorogenic steroids. The substance responsible for this fluorescence was subsequently shown by Finkelstein, von Euw, and Reichstein (27) to be pregnanetriol-11-one. During the control period no fluorogenic substance could be detected, whereas the equivalent of $6 \mathrm{mg}$. was estimated in the pooled urines during the treatment period.

Another new metabolite of 21-desoxyhydrocortisone was identified in the urine of both patients and was shown to be $3 \alpha, 11 \beta, 17 \alpha$-trihydroxypregnan-20-one, (isolated as its $\Delta^{9}$ analogue) (34).

\section{DISCUSSION}

The data presented above indicate that $17 \alpha-$ hydroxyprogesterone is metabolized to the 17 ketosteroids, etiocholanolone and androsterone.

$3 \alpha$-Hydroxy-pregnan-20-one and $17 \alpha$-hydroxypregnanolone have also been identified as metabolites of $17 \alpha$-hydroxyprogesterone, whereas the presence of pregnanetriol and pregnanediol has been indicated by means of their absorption spectra in sulfuric acid. The administration of 21-desoxyhydrocortisone, on the other hand, results in the appearance in the urine of the following 17 -ketosteroids : $11 \beta$-hydroxyandrosterone, 11 keto-androsterone, 11-keto-etiocholanolone, $11 \beta$ hydroxy-etiocholanolone. In addition, 11-ketopregnanolone, 21-desoxy-tetrahydrocortisone and 21-desoxy-tetrahydro-hydrocortisone were found. The possible presence of pregnanetriol-11-one was suggested by the fluorescent technique of Zondek and Finkelstein (33). Figures 1 and 2 illustrate these steroidal conversions.

The finding of pregnanolone-3 $\alpha$ and 11-ketopregnanolone as metabolites of $17 \alpha$-hydroxyprogesterone and 21-desoxyhydrocortisone respectively demonstrates the body's ability to dehydroxylate at the $\mathrm{C}-17$ position. The implication of these results will be more fully discussed in a subsequent publication (35).

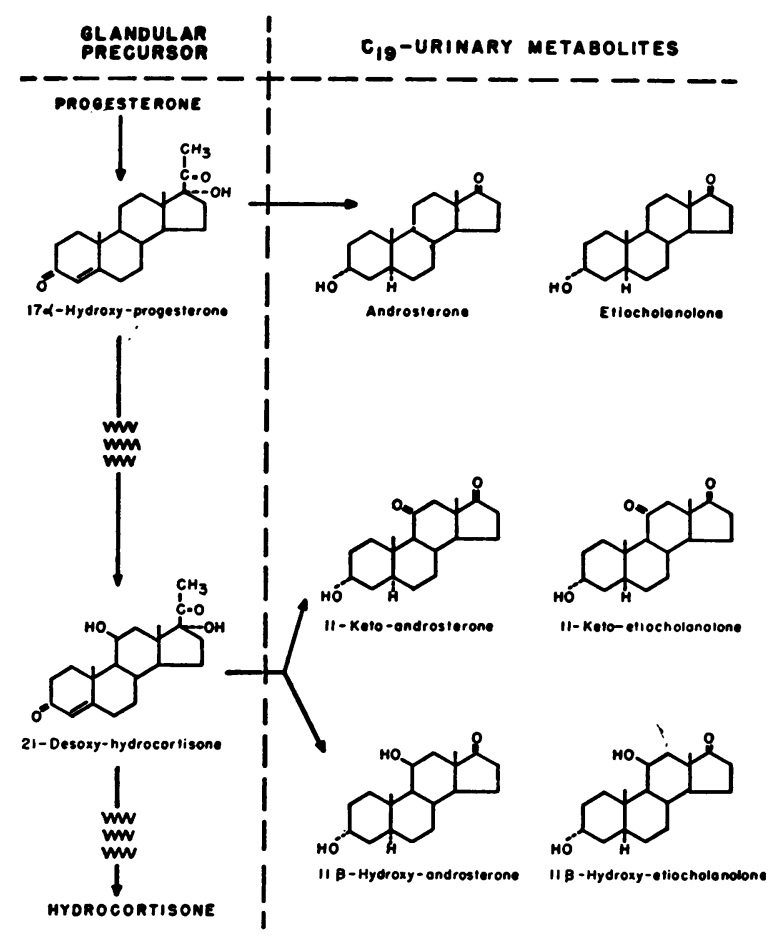

Fig. 1. The Urinary C-19 Metabolites of 17 $\alpha$-HyDROXYPROGESTERONE AND 21-DESOXYHYDROCORTISONE

Since the administration of $17 \alpha$-hydroxyprogesterone and 21-desoxyhydrocortisone produces a urinary steroidal pattern somewhat similar to that found in patients with congenital adrenal hyperplasia (compare Tables I with Figures 1 and 2) the possibility exists that these glandular products play an important role in this disease. This becomes all the more possible since $17 \alpha-$ hydroxyprogesterone has been shown to serve as a direct precursor of hydrocortisone in various biosynthetic experiments. While this has not been reported for 21-desoxyhydrocortisone, Meyer, Rodgers, and Pincus (36) have demonstrated that 21-desoxycortisone may be hydroxylated to produce cortisone in vitro.

As has been noted earlier, patients with congenital adrenal hyperplasia respond differently from the normal when exogenous ACTH is administered in that they apparently do not produce additional amounts of hydrocortisone. The increases in urinary ketosteroids and in the pregnanediol-pregnanetriol complex that do follow the administration of the trophic hormone suggest that the adrenals of these patients are capable of 


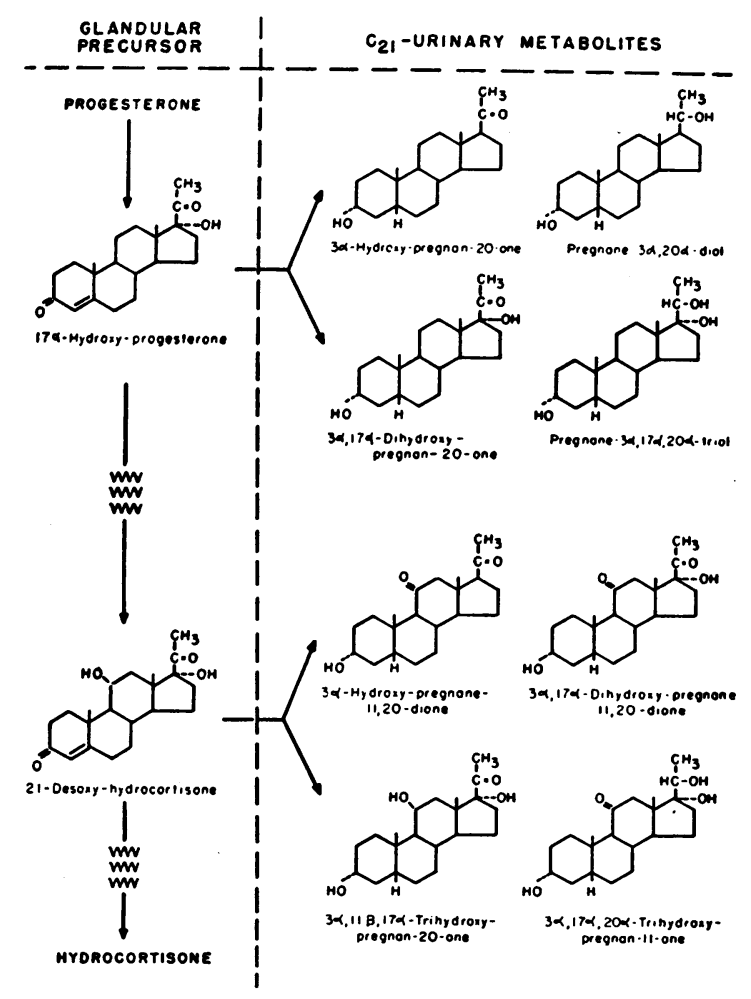

Fig. 2. The Urinary C-21 Metabolites of $17 \alpha$-HyDROXYPROGESTERONE AND 21-DESOXYHYDROCORTISONE

synthesizing adequately some of the precursors of hydrocortisone. The fact that the urinary metabolites found in congenital adrenal hyperplasia are structurally related to and in many cases identical with those found after the exogenous administration of $17 \alpha$-hydroxyprogesterone and 21-desoxyhydrocortisone indicates that the inability of such adrenals to synthesize hydrocortisone may be due to some defect in the enzymatic hydroxylation mechanisms which are necessary for the complete biosynthesis of hydrocortisone. A specific enzymatic defect in either the $11 \beta$ hydroxylase or the 21-hydroxylase systems has been implicated in adrenal hyperplasia $(12,37)$. However, the patterns of urinary steroid excretion given in Table I do not seem to indicate a single cause for this syndrome but rather they point to several individual defects, any of which could result in a failure to biosynthesize hydrocortisone in sufficient amounts. The absence of excessive amounts of 11-oxygenated C-19 metabolites in some patients (J. B., R. E., S. P., and J. L.) would suggest that the $11 \beta$-hydroxylation mechanisms in these patients is the main deficiency. In those patients (R. K., D. P., and E. B.) wherein large amounts of these metabolites were isolated, the principal block may be in the C-21 hydroxylation mechanism. Likewise, the isolation of large amounts of 11-keto-pregnanolone (L. Y.) also points to a failure of C-21 hydroxylation and possibly in addition to an inability to hydroxylate at C-17. Relative failure of this latter enzymatic process may also account for the isolation of excessive amounts of pregnanolone (R. K. and L. Y.). Bongiovanni and Eberlein (38) have presented evidence which indicates that in some children with adrenogenital syndrome hydroxylation at C-21 is not impaired.

The evidence accumulated to date does not appear to indicate that congenital adrenal hyperplasia is characterized by a single enzymatic block. Rather it seems likely that the similarity in all cases is a defect in hydroxylation; in some cases this occurs with those enzymes which hydroxylate at $\mathrm{C}-11$, in others with those systems responsible for hydroxylation at C-21, or still others with the C-17 hydroxylating mechanism. There may be instances where the $3 \beta$-hydroxylase ( $3 \beta$-hydroxy $-\Delta^{4}$-3-ketone) may be impaired and this could account for the large amounts of dehydroisoandrosterone occasionally found (D. P. and J. L.). In general, any event which results in diminished production of the end product, Compound F, leads to an accumulation of partially hydroxylated intermediates which upon escaping from the adrenal gland are metabolized along well-known paths. Some of the intermediates or their metabolites may be androgenic and could therefore be reponsible for the virilism observed. It should be pointed out that the virilism of patients with congenital adrenal virilism differs somewhat from that observed in patients with virilizing adrenal tumors or patients receiving large amounts of testosterone. Although the former patients do exhibit axillary and pubic hair together with an enlarged phallus or clitoris, body hirsutism is not usually encountered. The women may even have a female pubic escutcheon and they rarely have to shave. This is in contrast to the latter patients who exhibit marked body hirsutism.

In addition to enzymatic failures, Dorfman (39) has proposed that the virilism of the adrenogenital syndrome is due to the synthesis of large 
amounts of C-19 androgens, e.g., $\Delta^{4}$-androstene3,17-dione, etc., by the adrenals and that these arise from acetate or cholesterol but not via C-21 precursors, as, for example, pregnenolone. According to this hypothesis the synthesis of these adrenal androgens are interrelated with the adrenal secretion of the C-21 steroids since both processes are stimulated by the excessive amounts of ACTH thought to be present in the blood of such patients. It is difficult to explain by this concept the normal urinary 17 -ketosteroid values often observed in patients with Cushing's syndrome also due to bilateral adrenal hyperplasia (40). Moreover, as Bongiovanni and Clayton (32) have pointed out, the excretion of abnormal amounts of the C-21 steroid, pregnane-3 $\alpha, 17 \alpha$, $20 \alpha$-triol is more characteristic of adrenal hyperplasia than is an elevated 17-ketosteroid level.

The virilism due to adrenal tumors, on the other hand, may be considerably different in nature since the principal urinary steroid found in these instances is dehydroisoandrosterone whose metabolic precursors are still uncertain (41).

\section{SUMMARY}

An attempt to reproduce the urinary steroidal pattern characteristic of congenital adrenal virilism by means of the administration of $17 \alpha$-hydroxyprogesterone and 21-desoxyhydrocortisone has been made. The urinary metabolites of these steroids have been identified as: etiocholanolone; androsterone; 11-keto-etiocholanolone; 11-hydroxy-etiocholanolone; $11 \beta$ hydroxyandrosterone; pregnanolone; 17-hydroxy-pregnanolone and 11keto-pregnanolone. The presence of pregnanediol, pregnanetriol and pregnanetriol-11-one have been indicated by means of chromogenic reactions.

The following hypothesis is presented: Congenital adrenal hyperplasia is accompanied by enzymatic defects in the hydroxylation mechanisms, which results in the piling up of partially hydroxylated hydrocortisone precursors. These escape into the circulation and are metabolized along well-known pathways resulting in the production of androgens.

\section{REFERENCES}

1. Lewis, R. A., and Wilkins, L., The effect of adrenocorticotrophic hormone in congenital adrenal hyperplasia with virilism and in Cushing's syndrome treated with methyl testosterone. J. Clin. Invest., 1949, 28, 394.

2. Bartter, F. C., Albright, F., Forbes, A. P., Leaf, A., Dempsey, E., and Carroll, E., The effects of adrenocorticotropic hormone and cortisone in the adrenogenital syndrome associated with congenital adrenal hyperplasia: An attempt to explain and correct its disordered hormonal pattern. J. Clin. Invest., 1951, 30, 237.

3. Jailer, J. W., Louchart, J., and Cahill, G. F., Adrenal virilism. II. Metabolic studies. J. Clin. Invest., 1952, 31, 880.

4. Kelley, V. C., Ely, R. S., and Raile, R. B., Metabolic studies in patients with congenital adrenal hyperplasia. Effects of cortisone therapy. J. Clin. Endocrinol., 1952, 12, 1140.

5. Bayliss, R. I. S., Broadbent, I. E., and Steinbeck, A. W., Congenital adrenal hyperplasia. Effects of corticotrophin and tetrahydrocortisone. Lancet, 1954, 1, 434.

6. Bongiovanni, A. M., Eberlein, W. R., and Cara, J., Studies on the metabolism of adrenal steroids in the adrenogenital syndrome. J. Clin. Endocrinol. \& Metab., 1954, 14, 409.

7. Christy, N. P., Wallace, E. Z., and Jailer, J. W., The effect of intravenously-administered ACTH on plasma 17,21-dihydroxy-20-ketosteroids in normal individuals and in patients with disorders of the adrenal cortex. J. Clin. Invest., 1955, 34, 899.

8. Wilkins, L., Lewis, R. A., Klein, R., and Rosemberg, E., The suppression of androgen secretion by cortisone in a case of congenital adrenal hyperplasia. Bull. Johns Hopkins Hosp., 1950, 86, 249.

9 .Jailer, J. W., Louchart, J., and Cahill, G. F., Adrenal virilism. I. Diagnostic considerations and treatment. J. A. M. A., 1952, 150, 575.

10. Bastenie, P. A., Franckson, J. R. M., Verbiest, M., Kovacs, L., and Flamand, C. H., Cortisone treatment in adrenogenital syndrome. Lancet, 1953, 1, 915.

11. Sydnor, K. L., Kelley, V. C., Raile, R. B., Ely, R. S., and Sayers, G., Blood adrenocorticotrophin in children with congenital adrenal hyperplasia. Proc. Soc. Exper. Biol. \& Med., 1953, 82, 695.

12. Jailer, J. W., Virilism. Bull. New York Acad. Med., 1953, 29, 377.

13. Hechter, O., Zaffaroni, A., Jacobsen, R. P., Levy, H., Jeanloz, R. W., Schenker, V., and Pincus, G., The nature and the biogenesis of the adrenal secretory product. Recent Prog. in Hormone Res., 1951, 6, 215.

14. Hayano, M., and Dorfman, R. I., The action of adrenal homogenates on progesterone, 17-hydroxyprogesterone and 21-desoxycortisone. Arch. Biochem. \& Biophys., 1952, 36, 237.

15. Hechter, O., Biogenesis of adrenal cortical hormones. Ciba Foundation Coll. on Endocrinol., 1953, 7, 161.

16. Saba, N., Hechter, O., and Stone, D., The conversion of cholesterol to pregnenolone in bovine adrenal homogenates. J. Am. Chem. Soc., 1954, 76, 3862. 
17. Solomon, S., Lenz, A. L., Vande Wiele, R., and Lieberman, S., Pregnenolone, an intermediate in the biosynthesis of progesterone and the adrenocortical hormones. Abst. Am. Chem. Soc. 126th Meeting, 1954, p. 29C.

18. Pfiffner, J. J., and North, H. B., 17 $\beta$-hydroxyprogesterone. J. Biol. Chem., 1940, 132, 459.

19. Gassner, F. X., Personal communication.

20. Del Greco, F., Masson, G. M. C., and Corcoran, A. C., Effects of $\mathrm{C}_{17}$-hydroxy-steroids on urinary 17ketosteroids in the rat. Proc. Soc. Exper. Biol. \& Med., 1952, 80, 354.

21. Polley, H. F., and Mason, H. L., Rheumatoid arthritis: Effects of certain steroids other than cortisone and of some adrenal cortex extracts. J. A. M. A., 1950, 143, 1474.

22. Fukushima, D. K., Dobriner, K., and Gallagher, T. F., Metabolic studies with deuterium steroid hormones. Federation Proc., 1951, 10, 185.

23. Bongiovanni, A. M., Eberlein, W. R., and Cara, J., Studies on the metabolism of adrenal steroids in the adrenogenital syndrome. J. Clin. Endocrinol. \& Metab., 1954, 14, 409.

24. Butler, G. C., and Marrian, G. F., The isolation of pregnane-3,17,20-triol from the urine of women showing the adrenogenital syndrome. J. Biol. Chem., 1937, 119, 565.

25. Burstein, S., Savard, K., and Dorfman, R. I., The in vivo metabolism of 21-desoxycortisone. Endocrinology, 1953, 53, 267.

26. Dorfman, R. I., and Ungar, F., Metabolism of Steroid Hormones. Minneapolis, Minn., Burgess Pub. Co., 1953.

27. Finkelstein, M., v. Euw, J., and Reichstein, T., Isolierung von $3 \alpha, 17,20 \alpha$ Trioxy-pregnanon-(11) aus pathologischem menschlichem Harn. Helvet. chem. acta, 1953, 36, 1266.

28. Holtorff, A. F., and Koch, F. C., The colorimetric estimation of 17-ketosteroids and their application to urine extracts. J. Biol. Chem., 1940, 135, 377.

29. Sommerville, I. F., Marrian, G. F., and Kellar, R. J., Rapid determination of urinary pregnanediol. Lancet, 1948, 2, 89.

30. Dingemanse, E., Huis in 't Veld, L. G., and Hartogh-
Katz, S. L., Clinical method for the chromatographic-colorimetric determination of urinary 17ketosteroids. II. Normal adults. J. Clin. Endocrinol., 1952, 12, 66.

31. Lakshmanan, T. K., and Lieberman, S., An improved method of gradient elution chromatography and its application to the separation of urinary ketosteroids. Arch. Biochem. \& Biophys., 1954, 53, 258.

32. Bongiovanni, A. M., and Clayton, G. W., Jr., A simplified method for the routine determination of pregnanediol and pregnanetriol in urine. Bull. Johns Hopkins Hosp., 1954, 94, 180.

33. Zondek, B., and Finkelstein, M., A neutral fluorogenic substance in human urine and its increased excretion in pregnancy and pseudohermaphroditism. Acta Endocrinol., 1952, 11, 297.

34. Rosselet, J. P., Jailer, J. W., and Lieberman, S., Metabolism of $\mathrm{C}_{21}-17$ hydroxylated steroids. Federation Proc., 1955, 14, 272.

35. Rosselet, J.-P., Furman, M., Lieberman, S., and Jailer, J. W., In vivo conversion of $\mathrm{C}_{21}-17 \alpha$-hydroxylated steroid to $\mathrm{C}_{21}$-17-desoxymetabolites. Science, 1954, 120, 788.

36. Meyer, A. S., Rodgers, O. G., and Pincus, G., Cow adrenal perfusion of 21-desoxycortisone. Acta Endocrinologica, 1954, 16, 293.

37. Jailer, J. W., Chromatographic studies of urinary 17-ketosteroids in children with hyperadrenocorticism. 13th M \& R Pediatric Research Conf. Nov. 3 and 4, 1954, p. 33.

38. Bongiovanni, A. M., and Eberlein, W. R., The intermediary steroid metabolism in children with the adrenogenital syndrome. 13th $M$ \& $R$ Pediatric Res. Conf. Nov. 3 and 4, 1954, p. 79.

39. Dorfman, R. I., The adrenogenital syndrome. 13th M \& R Pediatric Research Conf. Nov. 3 and 4, 1954, p. 66.

40. Forbes, A. P., and Albright, F., A comparison of the 17-ketosteroid excretion in Cushing's syndrome associated with adrenal tumor and with adrenal hyperplasia. J. Clin. Endocrinol., 1951, 11, 926.

41. Lieberman, S., Origin of dehydroisoandrosterone. 13th M \& R Pediatric Res. Conf. Nov. 3 and 4, 1954 , p. 107. 